\title{
Anti-inflammatory effect of resveratrol on adjuvant arthritis rats with abnormal immunological function via the reduction of cyclooxygenase- 2 and prostaglandin $E_{2}$
}

\author{
XIAOYU CHEN, JINSENG LU, MEI AN, ZHONGFEI MA, HEXIANG ZONG and JUN YANG \\ Department of Histology and Embryology, Anhui Medical University, Hefei, Anhui 230032, P.R. China
}

Received January 10, 2014; Accepted March 7, 2014

DOI: $10.3892 / \mathrm{mmr} .2014 .2070$

\begin{abstract}
Rheumatoid arthritis (RA) is a chronic inflammatory disease with unknown etiology. The present study investigated the anti-inflammatory effect of resveratrol on rats with adjuvant arthritis (AA) with abnormal immunological function via the reduction of cyclooxygenase-2 (COX-2) and prostaglandin $\mathrm{E}_{2}\left(\mathrm{PGE}_{2}\right)$. AA model rats were established by injection of complete Freund's adjuvant and alterations in the rats secondary paw swelling and the polyarthritic scores were observed. Pathological examination of joint tissues was observed by hematoxylin and eosin staining. The proliferation of spleen cells was examined using a 3-(4,5-dimethylthiazol-2-yl)-2,5-diphenyltetrazolium bromide assay in vitro. The protein expression of COX-2 in the synovial tissues was detected by western blotting. The level of $\mathrm{PGE}_{2}$ in the serum was assayed using an ELISA kit. The results demonstrated that resveratrol (10 or $50 \mathrm{mg} / \mathrm{kg}$ ) was able to significantly reduce paw swelling and decrease the arthritis scores. Compared with the AA model rats, a significant reduction in the proliferation of concanavalin A-stimulated spleen cells was observed, articular cartilage degeneration with synovial hyperplasia and inflammatory cell infiltration was suppressed and the production of COX-2 and $\mathrm{PGE}_{2}$ in AA rats was reduced by treatment with resveratrol. These results suggest that resveratrol has significant anti-inflammatory effects on AA rats, which may be associated with the reduction of COX-2 and $\mathrm{PGE}_{2}$ inflammatory mediators.
\end{abstract}

Correspondence to: Dr Xiaoyu Chen, Department of Histology and Embryology, Anhui Medical University, 81 Meishan Road, Shushan, Hefei, Anhui 230032, P.R. China

E-mail: cxyayd@163.com

Abbreviations: SD rat, Sprague-Dawley rat; RA, rheumatoid arthritis; AA, adjuvant arthritis; COX, cyclooxygenase; $\mathrm{PGE}_{2}$, prostaglandin $\mathrm{E}_{2}$; NSAID, non-steroidal anti-inflammatory drug; $\mathrm{H} \& \mathrm{E}$, hematoxylin and eosin staining; con A, concanavalin A

Key words: adjuvant arthritis, resveratrol, immune, inflammation, rat

\section{Introduction}

Rheumatoid arthritis (RA) is a chronic inflammatory disease with an unknown etiology leading to cartilage and bone erosion and has a severe impact on human health and quality of life $(1,2)$. At present, the pathological characteristics of RA mainly include joint inflammation of the synovial tissue and excessive hyperplasia (3). The variation in hematology and joint histopathology of adjuvant arthritis (AA) rats, which is a commonly used animal model, is similar to human RA (4). Resveratrol, a natural plant flavone, which is abundantly present in grapes, fruit, red wine and other food products and medicinal plants, possesses pharmacological effects, including immune-regulatory, anti-inflammatory, antioxidant and antitumor activity (5-7). Resveratrol has been demonstrated to inhibit the enzymatic activity of cyclooxygenase (COX)-1 and COX-2, which are important in the pathogenesis of RA $(8,9)$. $\mathrm{COX}$ is a key rate-limiting enzyme of prostaglandin (PG) production in organisms. It has previously been reported that resveratrol is able to inhibit several experimental autoimmune diseases $(10,11)$. Based on previous studies concerning resveratrol and its anti-arthritic activity the anti-inflammatory mechanism of resveratrol on the animal model of AA rats, which involves COX-2 and PGE2 associated with RA is remains inadequate $(12,13)$. However, the detailed mechanisms underlying the protective effects of resveratrol on arthritis remain to be fully elucidated. In addition, oral administration of resveratrol (10 or $50 \mathrm{mg} / \mathrm{kg}$ body weight) over a period of two weeks reversed arthritis dysfunction in AA rats $(12,14)$. The aim of the present study was to detect the anti-inflammatory effects of resveratrol in an AA rat model and to determine the underlying mechanism of action.

\section{Materials and methods}

Animals. Male Sprague-Dawley (SD) rats (weight, 200 $\pm 20 \mathrm{~g}$; age, 8-10 weeks; certificate no., 2013-0002) were obtained from the Laboratory Animal Center of Anhui Medical University (Hefei, China). All experimental procedures were approved for the use of animals in research by the Ethics Review Committee for Animal Experimentation (Anhui Medical University). The animals were housed in standard laboratory conditions and fed ad libitum with a controlled ambient temperature of $22 \pm 2^{\circ} \mathrm{C}$ and a humidity of 50-60\%. A $12 \mathrm{~h}$ light/dark cycle was main- 
tained at all times. The rats were housed with five animals per cage, acclimated to the housing conditions and handled for 1 week prior to experiments.

Reagents. Resveratrol (purity, 99\%) was purchased from Sigma (St. Louis, MO, USA) and was dissolved in dimethylsulfoxide (DMSO). Celecoxib, which was also dissolved in DMSO, was purchased from Shanghai Pharmaceutical Co., Ltd. (Shanghai, China). Celecoxib is widely used in the treatment of RA as a selective COX-2 inhibitor and as positive drugs (15). Dulbecco's modified Eagle's medium (DMEM) was purchased from HyClone Laboratories, Inc. (Logan, UT, USA). All other reagents were of analytical purity. ELISA kits for $\mathrm{PGE}_{2}$ were purchased from Research \& Development Systems, Inc. (Minneapolis, MN, USA). A stock solution was prepared in DMSO and the final concentration of DMSO with diluted phosphate-buffered saline (PBS) was $0.05 \%(\mathrm{w} / \mathrm{v})$. Rabbit polyclonal anti-COX-2 antiserum was obtained from Santa Cruz Biotechnology, Inc. (Santa Cruz, CA, USA) and concanavalin A (con A) was purchased from Sigma.

\section{Methods}

Induction of $A A$. AA model rats were induced as previously described (16,17). Briefly, complete Freund's adjuvant (CFA) was purchased from Sigma and suspended in heat-killed bacillus Calmette-Guerin (Shanghai Biochemical Institute, Shanghai, China) in liquid paraffin at $10 \mathrm{mg} / \mathrm{ml}$. Arthritis was induced in SD rats by intradermal injection of $0.1 \mathrm{ml} \mathrm{CFA}$ emulsion into the right hind metatarsal footpad. The normal control rats were intradermally injected with $0.1 \mathrm{ml}$ liquid paraffin into the right hind feet pads.

Treatment of $A A$. The rats were randomly divided into the following five groups ( $n=10$ per group): The normal group, AA model group, AA rats which were administered resveratrol via continuous intragastric gavage (10 or $50 \mathrm{mg} / \mathrm{kg}$, daily) and rats treated with celecoxib (5 mg/ $\mathrm{kg}$, every day) between day 12 and day 28 after immunization. The normal and AA model rat groups were subcutaneously administered the same volume of $(0.05 \%, \mathrm{w} / \mathrm{v})$ the vehicle (DMSO) for the same time period.

Assessment of arthritis. Clinical assessments were performed by two independent observers who had no knowledge of the treatment protocol. AA severity was evaluated by the previously described scoring system $(18,19)$. The clinical parameters of non-injected swelling (left hind paw of rats) and the polyarthritis index of the AA rats were evaluated every four days between day 12 and day 28 after immunization. The left hind paw volume (ankle joint) was assessed using a water replacement plethysmometer (Shandong Academy of Medical Science, Jinan, China), the degree of swelling $(\Delta \mathrm{ml})=$ after inflammatory volume - former non-inflammatory volume. The polyarthritic scale in each paw was graded on a 0-4 scale as follows: 0, normal; 1 , paws in one joint with redness and/or swelling; 2, paws in less than one joint with mild redness and/or swelling; 3, all paws with severe redness and/or swelling; and 4 , paws with deformity and/or ankylosis. The cumulative score for all three non-injected paws of each rat was used as the polyarthritis index with a maximum value of 12 .
Histological examination with hematoxylin and eosin $(H \& E)$ staining. Animals were anesthetized by inhalation of $2.5-4 \%$ isoflurane (Shanghai Mindray, Shanghai, China) on the day 28, and sacrificed immediately by exsanguination. The secondary hind paws were removed above the ankle joints and were fixed in $4 \%$ formaldehyde at $4{ }^{\circ} \mathrm{C}$ overnight, and then decalcified in ethylenediaminetetraacetic acid buffer for 4 weeks prior to dehydration and paraffin embedding. The serial sections $(3 \mu \mathrm{m})$ were stained with $H \& E$ to microscopically examine (Olympus Corporation, Tokyo, Japan) cell infiltration, pannus formation, synovial hyperplasia, cartilage damage and bone erosion. The pathology of the joint was scored as previously described (20) using the following scoring system: Cellular infiltration, synovial proliferation, cartilage erosion and pannus formation, graded between 0 (no infiltration/normal synovium/no changes/no abnormalities) and 4 (extensive infiltrates invading the joint capsule with maximal cellular influx/severe synovial hyperplasia and effacement of joint space and adjacent cartilage and bone/extensive deep cartilage degradation/extensive pannus formation, infiltration, flat overgrowth of the joint surface). A mean score of the paws was calculated. Evaluation of the joint pathology of the tissue specimen was repeated five times by two independent observers.

\section{3-(4,5-dimethylthiazol-2-yl)-2,5-diphenyltetrazolium bromide} (MTT) assay for lymphocyte proliferation. Lymphocyte proliferation was determined using an MTT (Sigma) assay (21). The rats were anaesthetized and sacrificed on day 28 in each group. The spleens were immediately removed under sterile conditions and gently crushed with a syringe in DMEM. Erythrocytes were filtered on a 100-mesh sieve and then washed and resuspended in DMEM. The cell suspension $(100 \mu \mathrm{l})$ was incubated in 96-well culture plates (Corning Inc., Corning, NY, USA) with $5 \times 10^{6}$ cells $/ \mathrm{ml}$ and six wells per sample. DMEM $(100 \mu \mathrm{l})$ containing $10 \%$ fetal bovine serum was added to three wells (controls) and cell culture medium in the other three wells was treated with Con A (at a final concentration of $5 \mu \mathrm{g} / \mathrm{ml}$ ). Spleen lymphocytes were incubated at $37^{\circ} \mathrm{C}$ in a $5 \% \mathrm{CO}_{2}$ air atmosphere. Following culture for three days, $100 \mu \mathrm{l}$ supernatant was discarded and $20 \mu \mathrm{l}$ MTT (final concentration of $5 \mathrm{mg} / \mathrm{ml}$ ) was added to each well and oscillated for $1 \mathrm{~min}$ on an oscillator, and incubated for $4 \mathrm{~h}$. Following incubation, the cultures were centrifuged (760 x g; $10 \mathrm{~min})$. Then, $150 \mu \mathrm{l}$ DMSO (Sigma) was added to each well. The absorbance (A) was measured on a Microplate Reader Model 550 (Bio-Rad, Hercules, CA, USA) at $570 \mathrm{~nm}$. The results were described as an average of $\mathrm{A}$ and the experiments were repeated in triplicate.

Protein expression of $\mathrm{COX}-2$ detected by western blotting in synovial tissues. COX-2 protein expression was analyzed by western blotting. The rats were sacrificed on day 28 in each group. The synovial tissues of the left ankle joints were removed, weighed and grinded. The lysates were sonicated for $1 \mathrm{~min}$ on ice and centrifuged at 7,500 $\mathrm{x} \mathrm{g}$ for $10 \mathrm{~min}$ to sediment the particulate material. The protein concentration of the supernatant was measured using a bicinchoninic acid assay kit (Agilent Technologies, Inc., Santa Clara, CA, USA). The protein $(50 \mu \mathrm{g})$ was separated by $10 \%$ sodium dodecyl sulfate-polyacrylamide gel electrophoresis. The resolved proteins were transferred onto a nitrocellulose membrane 

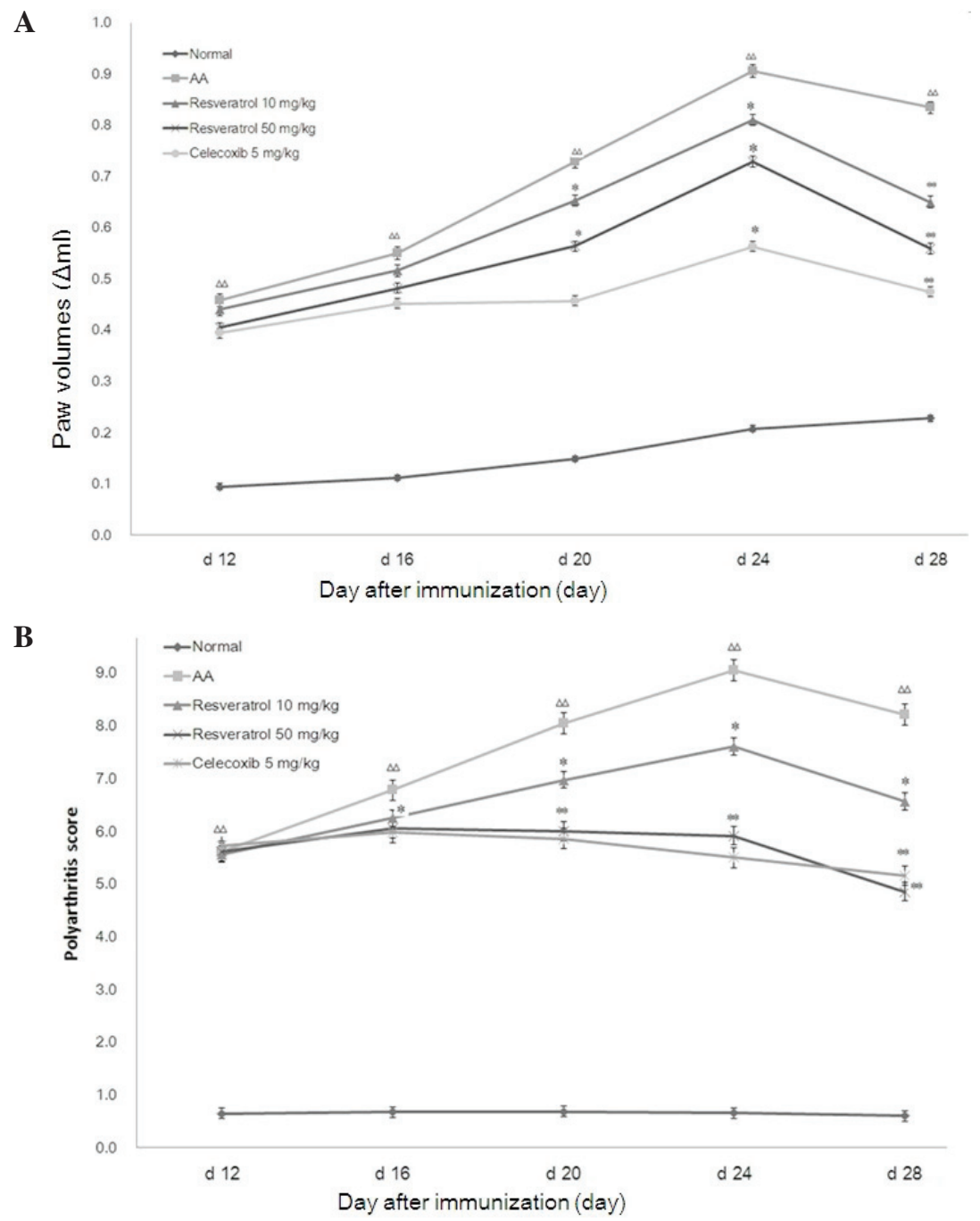

Figure 1. Effects of resveratrol (10 and $50 \mathrm{mg} / \mathrm{kg}$ ) and celecoxib (5 mg/kg) on (A) hind paw swelling and (B) polyarthritis scores in AA rats (mean \pm standard deviation; $\mathrm{n}=10) .{ }^{\Delta \Delta} \mathrm{P}<0.01$ compared with the normal group; ${ }^{*} \mathrm{P}<0.05$ and ${ }^{* *} \mathrm{P}<0.01$ compared with the model group. AA, adjuvant arthritis.

(Bio-Rad) and then incubated with primary antibodies (rabbit polyclonal anti-COX-2 antiserum 1:2,000 dilution and $\beta$-actin 1:5,000 dilution) for $1 \mathrm{~h}$ at room temperature and then at $4^{\circ} \mathrm{C}$ overnight. The membrane was washed three times using Tris-buffered saline with Tween-20 and secondary antibody to IgG (Santa Cruz Biotechnology, Inc.) conjugated to horseradish peroxidase for $2 \mathrm{~h}$ at room temperature. The blots were probed with the enhanced chemiluminescence western blotting substrate (Pierce Biotechnology, Inc., Rockford, IL, USA). The protein expression levels were normalized to $\beta$-actin.

$P G E_{2}$ levels in the serum determined by ELISA assays. On day 28 , the rats were sacrificed and the serum from peripheral blood was collected and stored at $-80^{\circ} \mathrm{C}$ prior to the assay. Concentrations of $\mathrm{PGE}_{2}$ in the serum were measured using ELISA kits according to the manufacturer's instructions. Each serum sample was assessed in triplicate.

Statistical analysis. The results are expressed as the mean \pm standard deviation, where $n$ indicates the number of rats. Analysis of variance was used in the SPSS version 17.0 professional software (SPSS, Inc., Chicago, IL, USA) to determine significant differences between the groups. The histological scores were analyzed using a non-parametric Mann-Whitney $\mathrm{U}$ test. $\mathrm{P} \leq 0.05$ was considered to indicate a statistically significant difference.

\section{Results}

Effects of resveratrol on the secondary inflammatory reaction in AA rats. SD rats in the AA groups developed typical clinical symptoms of severe arthritis (joint redness and swelling of the feet) and progressed rapidly. Compared with the normal rats, the onset of secondary arthritis (the left hind paw) significantly increased in AA rats on day 12 after immunization. Compared with the normal group, the weight of the rats in the model group markedly decreased and the left hind paw was markedly swollen. Resveratrol (10 or $50 \mathrm{mg} / \mathrm{kg}$ ) was able to relieve paw swelling $(\mathrm{P}<0.01)$ and polyarthritis from day 24 . Similar results were observed with celecoxib treatment (5 mg/kg; Fig. 1).

Effects of resveratrol on the histopathology of AA rats. On day 28 after immunization, histological features of the pathological microscopic findings were observed (Fig. 2). Joint 

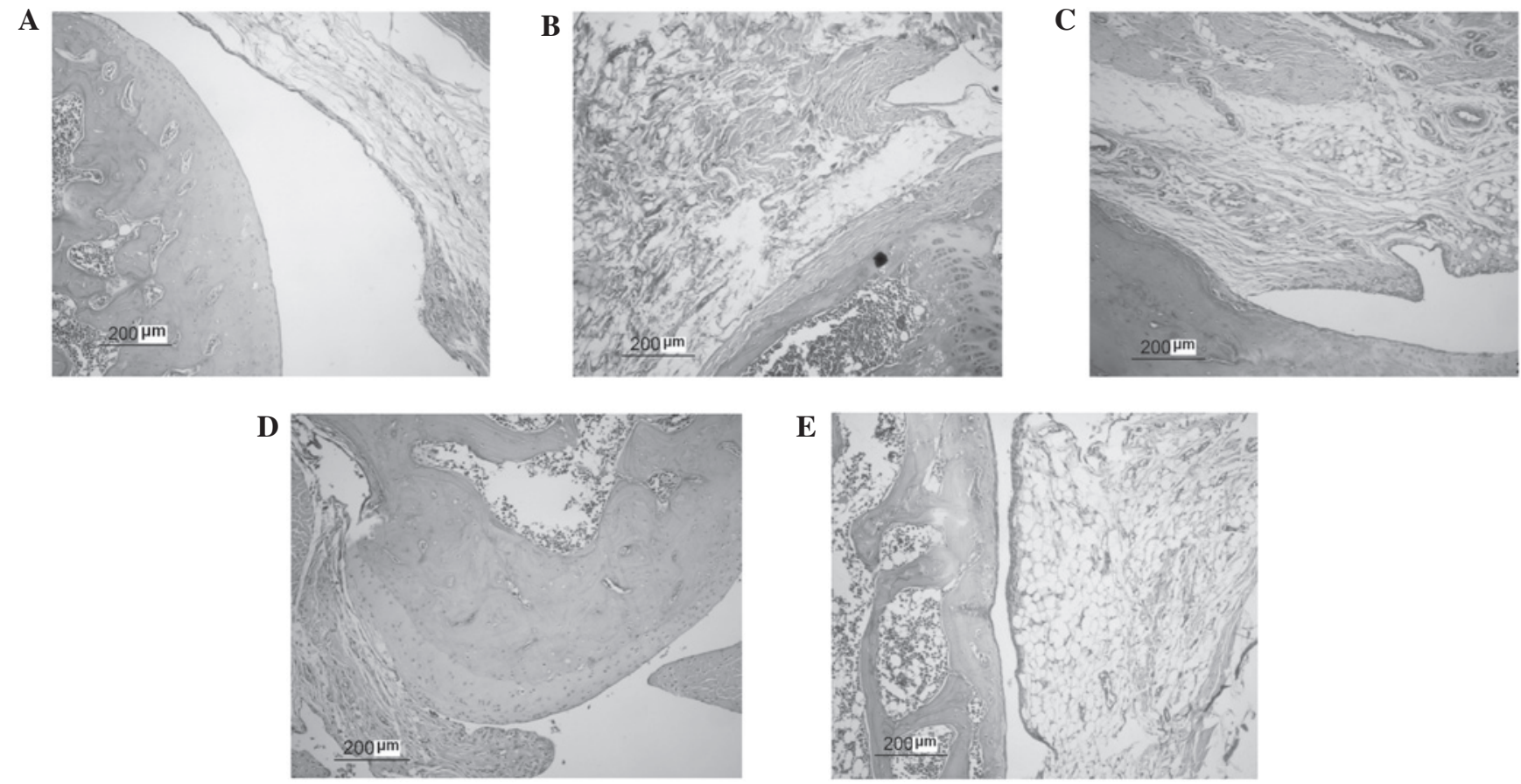

Figure 2. Histopathological features of representative ankle joints in Sprague-Dawley rats (hematoxylin and eosin staining; magnification, x100). (A) Articular cartilage with a normal appearance and a joint space. (B) Adjuvant arthritis model rats on day 30 after immunization with a narrow joint space and synovial hyperplasia. (C-D) Resveratrol (10 and $50 \mathrm{mg} / \mathrm{kg}$ ) and (E) celecoxib (5 mg/kg)-treated rats on day 28 after immunization.

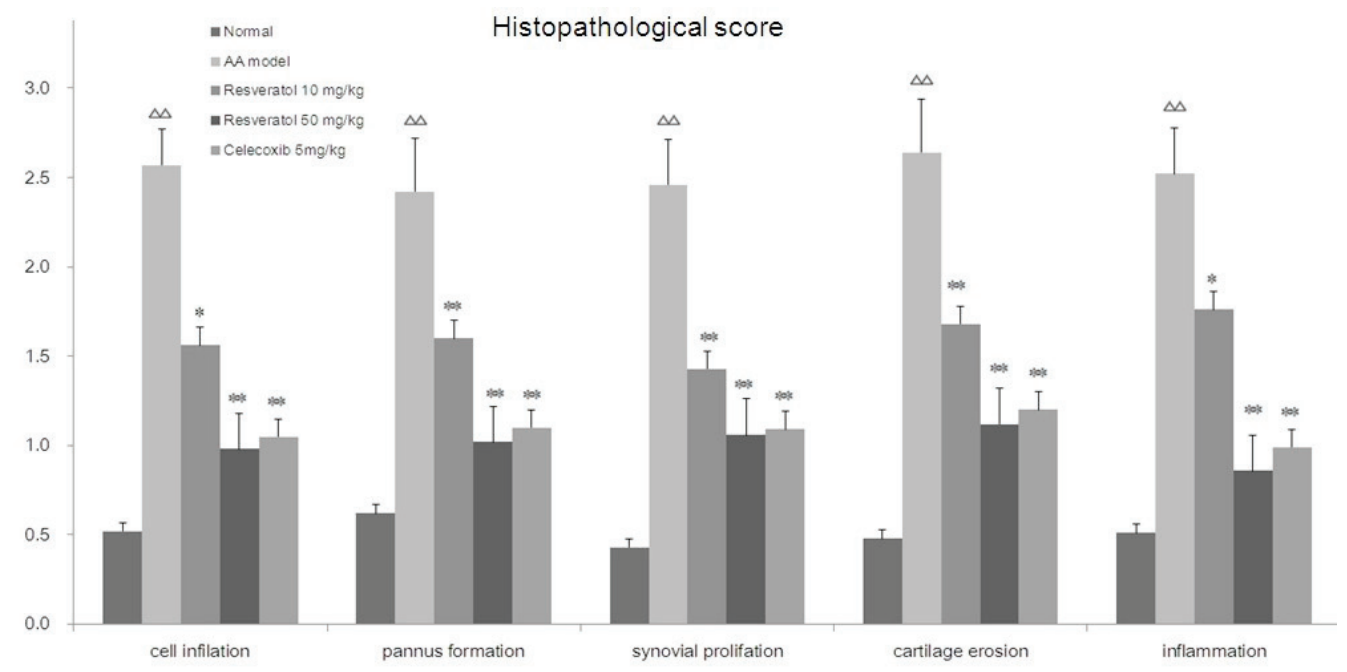

Figure 3. Histopathological evaluation of the ankle joint in adjuvant arthritis rats treated with resveratrol $\left(\mathrm{n}=5 ;\right.$ mean \pm standard deviation). ${ }^{\Delta \Delta} \mathrm{P}<0.01 \mathrm{compared}$ with the normal group; ${ }^{*} \mathrm{P}<0.05$ and ${ }^{* *} \mathrm{P}<0.01$ compared with the model group.

structure sections were stained with H\&E. In normal rats, the joint structures were clear, synoviocytes were monolayer and inflammatory cells had not infiltrated the articular cartilage. In AA model rats, the joint structure showed signs of severe arthritis, including synovial tissue hyperplasia, inflammatory cell infiltration in the synovial lining layer, novel blood vessel formation, and articular cartilage erosion and degradation. Resveratrol (10 or $50 \mathrm{mg} / \mathrm{kg}$ ) was able to inhibit synovial hyperplasia and pannus formation, reduce inflammatory cell infiltration and alleviate the destruction of articular cartilage. Celecoxib $(5 \mathrm{mg} / \mathrm{kg})$ had similar effects to resveratrol on the histopathology of AA rats. Histopathological scores for the presence of inflammatory cell infiltration, pannus formation, synovial proliferation, cartilage erosion and synovial inflammation of the ankle joints in AA rats, which were treated with resveratrol are shown in Fig. 3. Compared with the normal group, the histopathological scores in the AA rats significantly increased $(\mathrm{P}<0.01)$. Treatment with resveratrol significantly decreased histopathological scores compared with the AA rats $(\mathrm{P}<0.05, \mathrm{P}<0.01)$. The celecoxib-treated $(5 \mathrm{mg} / \mathrm{kg})$ group exhibited similar results to the group treated with resveratrol.

Effects of resveratrol on lymphocyte proliferation in AA rats. As shown in Fig. 4, compared with the normal group, con A-induced lymphocyte proliferation in the model group increased, while in the resveratrol group (10 or $50 \mathrm{mg} / \mathrm{kg}$ ) 


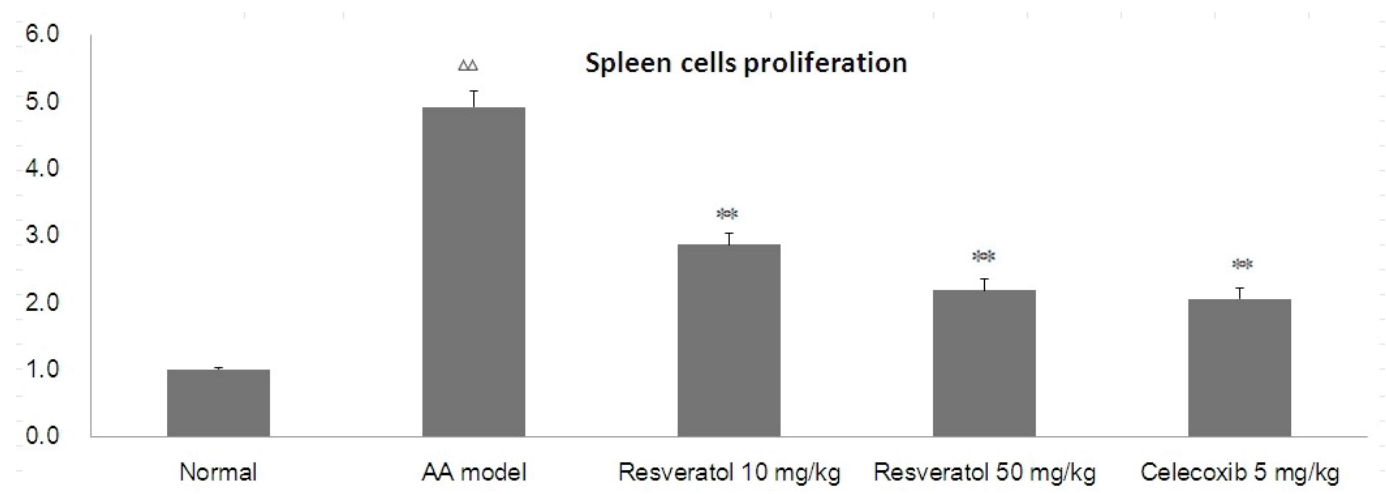

Figure 4. Effects of resveratrol on lymphocyte proliferation in adjuvant arthritis rats ( $\mathrm{n}=3$; mean \pm standard deviation). ${ }^{\Delta \Delta} \mathrm{P}<0.01$ compared with the normal group; ${ }^{* *} \mathrm{P}<0.01$ compared with the model group. AA, adjuvant arthritis.

A

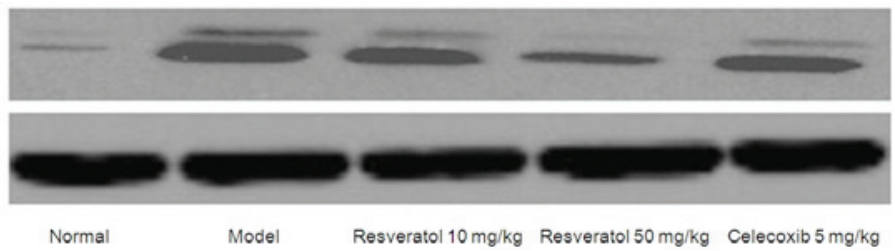

$\mathbf{B}$

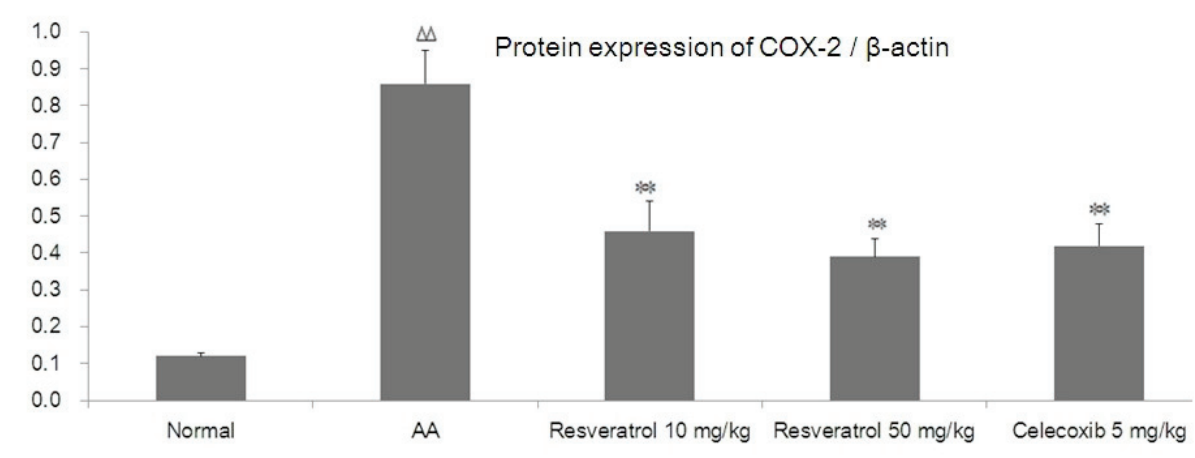

Figure 5. Effect of the different concentrations of resveratrol on the expression of COX-2 in AA synovial tissue. (A) Protein expression was assayed by western blotting. (B) Mean protein expression of COX-2/ $\beta$-actin quantified by scanning densitometry (mean \pm standard deviation; $\mathrm{n}=6$ ). ${ }^{\Delta \Delta} \mathrm{P}<0.01 \mathrm{vs}$. the normal group; ${ }^{* *} \mathrm{P}<0.01$ vs. the model group. AA, adjuvant arthritis; COX-2, cyclooxygenase- 2 .

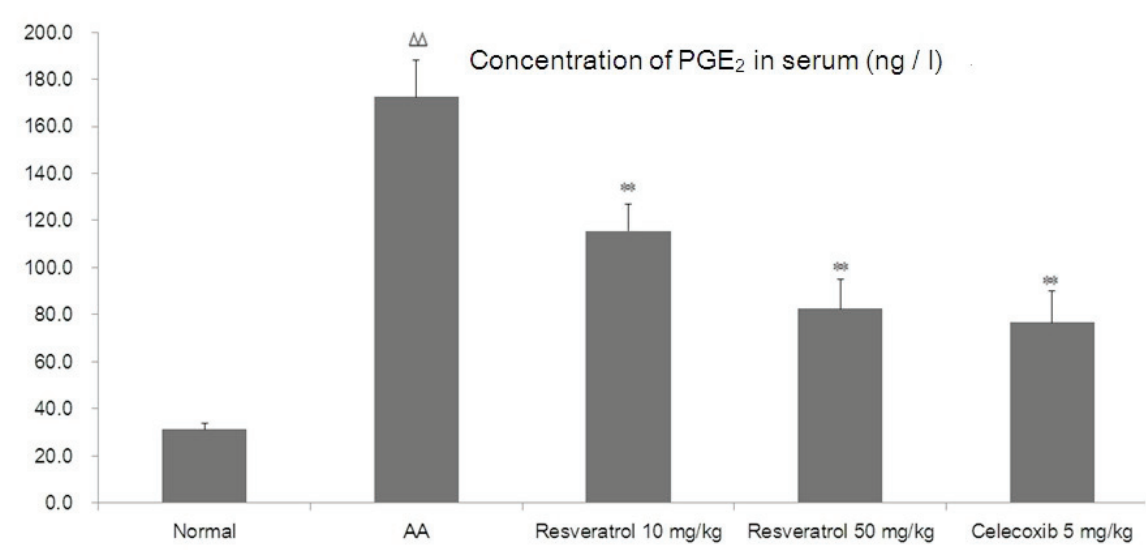

Figure 6. Resveratrol regulates the concentration of $\mathrm{PGE}_{2}$ in the serum (mean \pm standard deviation; $\mathrm{n}=3$ ). ${ }^{\Delta \Delta} \mathrm{P}<0.01$ vs. the normal group; ${ }^{*} \mathrm{P}<0.05$ and ${ }^{* *} \mathrm{P}<0.01$ vs. the model group. $\mathrm{PGE}_{2}$, prostaglandin $\mathrm{E}_{2} ; \mathrm{AA}$, adjuvant arthritis.

con A-induced lymphocyte proliferation was significantly inhibited. Celecoxib $(5 \mathrm{mg} / \mathrm{kg})$ also significantly decreased T-cell proliferation $(\mathrm{P}<0.01)$.
Effect of resveratrol on the protein expression of $\mathrm{COX}-2$ in the synovial tissues of AA rats. To investigate the potential mechanism underlying the actions of resveratrol on synovial 
hyperplasia, the protein expression of COX-2 was detected by western blot analysis (Fig. 5A). Compared with the normal group, a high level of COX-2 protein expression was detected in the AA model group. The resveratrol-treated group exhibited a significantly decreased COX-2 level in the synovial tissue. Celecoxib $(5 \mathrm{mg} / \mathrm{kg})$ also significantly reduced the level of COX-2 in synovial tissue. As shown in Fig. 5B, compared with the normal group, the protein expression of COX-2 significantly increased in AA rats $(\mathrm{P}<0.01)$. Treatment with resveratrol significantly decreased the expression of COX-2 compared with the AA rats $(\mathrm{P}<0.01)$. The celecoxib-treated $(5 \mathrm{mg} / \mathrm{kg})$ group demonstrated similar results to the group treated with resveratrol (Fig. 5B).

Resveratrol regulates the concentration of $P G E_{2}$ in the serum. As shown in Fig. 6, compared with the normal group, the concentrations of $\mathrm{PGE}_{2}$ significantly increased in the serum of AA rats $(\mathrm{P}<0.01)$. Treatment with resveratrol $(10$ or $50 \mathrm{mg} / \mathrm{kg}$ ) significantly decreased the concentrations of $\mathrm{PGE}_{2}$ compared with AA rats $(\mathrm{P}<0.05$ or $\mathrm{P}<0.01$, respectively). The celecoxib-treated group demonstrated similar results to the group treated with resveratrol.

\section{Discussion}

RA is a progressive inflammatory joint disease, which affects $\sim 1 \%$ of the population worldwide; however, the etiology of RA remains to be elucidated (1-3). AA is one of the most characterized animal models of RA, which provides substantial insights into basic pathogenic mechanisms and assesses potential novel drugs for the treatment of human RA. In the present study, SD rats were treated with complete Freund's adjuvant (CFA) to establish an AA model as previously described (4). Drugs, including resveratrol and celecoxib were administered via continuous intragastric gavage between day 12 and day 28 after immunization. For the normal and AA model groups, the rats were administered an equal quantity of DMSO solution.

The main pathohistological characteristics of RA include synovitis, inflammatory cell infiltration, pannus formation, synovial hyperplasia as well as cartilage and bone erosion. The polyphenol resveratrol (2,3,4'-trihydroxystilbene), a safe, well-described plant-derived compound, which is present in red wine, possesses cardiovascular benefits as well as anti-inflammatory and immune-regulatory properties. Several studies have demonstrated that resveratrol was able to suppress T-cell expansion and pro-inflammatory cytokine production in vivo and in vitro (22). In the present study, resveratrol was found to inhibit synovial hyperplasia and pannus formation, reduce inflammatory cell infiltration and alleviate the destruction of articular cartilage in AA rats as determined by histological examination and pathology scores.

RA is generally accepted to be a disorder of the immune system and lymphocytes are considered to be important in the pathogenesis of RA. For the treatment of RA, strategies have shifted from nonsteroidal anti-inflammatory drugs (NSAIDs) that inhibit the disease process, to the regulation of the immune system and biological agents (23). Although the specific mechanism by which $\mathrm{T}$ cells induce arthritis remains to be elucidated. Various subtypes of T cells have an important function in the complex inflammatory cell interaction network, which is directly associated with the development and outcome of the disease (24). In the present study, con A-induced lymphocyte proliferation increased in the model group compared with the normal group, while the resveratrol-treated group was able to significantly inhibit con A-induced lymphocyte proliferation. This suggested that the effect of resveratrol on adjuvant-induced arthritis in SD rats may be associated with T-cell immune regulation. The primary cause of RA is yet to be fully elucidated and the involvement of $\mathrm{T}$ cell-associated events early in rheumatoid synovitis remain controversial. Several studies using this animal model established that a variety of $\mathrm{T}$ cells were able to contribute to synovitis $(25,26)$. In the future, our aim is to further investigate the effects of resveratrol on T-cell function and the association between the cytokines, such as IL-1, TGF- $\beta$ and IL- 6 .

Cytokines are important in the pathogenesis of a wide variety of inflammatory and autoimmune diseases. COX is a key rate-limiting enzyme for $\mathrm{PG}$ production in animals. COX catalyzes the conversion of arachidonic acid into $\mathrm{PGH}_{2}$, which is further metabolized into various types of $\mathrm{PG}_{\mathrm{S}}(9)$. These $\mathrm{PG}_{\mathrm{S}}$ are involved in human physiology and pathophysiological processes. $\mathrm{PGE}_{2}$ is a vital inflammatory-disease mediator, which is important in inflammatory processes, including fervescence, edema and vascular permeability (8-10). Additionally, traditional NSAIDs exhibit anti-inflammatory effects and produce side effects that are associated with COX. Two COX isoforms have been identified, COX-1 and COX-2. COX-2 evokes $\mathrm{PGE}_{2}$ production and sustains inflammatory diseases (10). A highly selective COX-2 inhibitor is important in the clinical treatment of RA (27-29). COX-2 is an inflammatory mediator, which is highly expressed in the synovial tissue of patients with RA, and is involved in the joint inflammatory process (9-11). In addition, PGs, particularly $\mathrm{PGE}_{2}$, which is excessively expressed in RA, is important in synovial tissue vasodilation, liquid leakage and pain (30). The present study found a high expression level of COX-2 protein in synovial tissues and $\mathrm{PGE}_{2}$ in the serum of $\mathrm{AA}$ model rats. Resveratrol was able to reduce overexpression of the COX-2 protein in synovial tissues and concentrations of $\mathrm{PGE}_{2}$ in the serum.

In conclusion, $\mathrm{RA}$ is a disease with a complex pathogenesis that is currently difficult to treat. Adjuvant-induced arthritis, a T-cell-mediated chronic inflammatory disease, has been widely used as an RA model for polyarthritis in rats, and for identifying potential therapeutic targets (31). Resveratrol may represent a novel approach to the management of RA and associated syndromes (32). The present study revealed that resveratrol markedly improved arthritic histopathology in AA rats, which may be associated with the modification of the abnormal immunological function of AA rats, and may also be associated with the reduction of COX-2 and $\mathrm{PGE}_{2}$ inflammatory cytokines. The present study provides a basis for further investigation of the anti-inflammatory effect of resveratrol on AA.

\section{Acknowledgements}

This study was supported by grants from the National Natural Science Foundation of China (grant no. 81373421), the Innovative Entrepreneurial Training of the National College Students' Program (grant no. 201310366007) and the Natural 
Science Foundation of Higher Education Institutions of Anhui Province, China (grant no. KJ2010A183).

\section{References}

1. Karmakar S, Kay J and Gravallese EM: Bone damage in rheumatoid arthritis: mechanistic insights and approaches to prevention. Rheum Dis Clin North Am 36: 385-404, 2010.

2. Mattey DL, Glossop JR, Nixon NB and Dawes PT: Circulating levels of tumor necrosis factor receptors are highly predictive of mortality in patients with rheumatoid arthritis. Arthritis Rheum 56: 3940-3948, 2007.

3. Bax M, van Heemst J, Huizinga TW and Toes RE: Genetics of rheumatoid arthritis: what have we learned? Immunogenetics 63 : 459-466, 2011.

4. Chen XY, Li J, Cheng WM, Jiang H, Xie XF and Hu R: Effect of total flavonoids Chrysanthemum indicum on the apoptosis of synoviocytes in joint of adjuvant arthritis rats. Am J Chin Med 36: 695-704, 2008

5. Bereswill S, Muñoz M, Fischer A, Plickert R, Haag LM, Otto B, Kühl AA, Loddenkemper C, Göbel UB and Heimesaat MM Anti-inflammatory effects of resveratrol, curcumin and simvastatin in acute small intestinal inflammation. PLoS One 5 e15099, 2010.

6. Kavas GO, Ayral PA and Elhan AH: The effects of resveratrol on oxidant/ antioxidant systems and their cofactors in rats. Adv Clin Exp Med 22: 151-155, 2013.

7. Tyagi A, Gu M, Takahata T, Frederick B, Agarwal C, Siriwardana S, Agarwal R and Sclafani RA: Resveratrol selectively induces DNA Damage, independent of Smad4 expression, in its efficacy against human head and neck squamous cell carcinoma. Clin Cancer Res 17: 5402-5411, 2011.

8. Kundu JK, Shin YK, Kim SH and Surh YJ: Resveratrol inhibits phorbol ester-induced expression of COX-2 and activation of NF-kappaB in mouse skin by blocking IkappaB kinase activity Carcinogenesis 27: 1465-1474, 2006.

9. Yar AS, Menevse S and Alp E: The effects of resveratrol on cyclooxygenase- 1 and -2 , nuclear factor kappa beta, matrix metalloproteinase-9, and sirtuin 1 mRNA expression in hearts of streptozotocin-induced diabetic rats. Genet Mol Res 10 2962-2975, 2011.

10. Shindler KS, Ventura E, Dutt M, Elliott P, Fitzgerald DC and Rostami A: Oral resveratrol reduces neuronal damage in a model of multiple sclerosis. J Neuroophthalmol 30: 328-339, 2010.

11. Singh NP, Hegde VL, Hofseth LJ, Nagarkatti M and Nagarkatti P: Resveratrol (trans-3,5,4'-trihydroxystilbene) ameliorates experimental allergic encephalomyelitis, primarily via induction of apoptosis in T cells involving activation of aryl hydrocarbon receptor and estrogen receptor. Mol Pharmacol 72: 1508-1521, 2007.

12. Chen XY, Wang ZC, Li J, Liu XL and Sun YH: Regulation of synoviocyte activity by resveratrol in rats with adjuvant arthritis. Exp Ther Med 6: 172-176, 2013.

13. Tian J, Chen JW, Gao JS, Li L and Xie X: Resveratrol inhibits TNF- $\alpha$-induced IL-1 $\beta$, MMP-3 production in human rheumatoid arthritis fibroblast-like synoviocytes via modulation of PI3kinase/Akt pathway. Rheumatol Int 33: 1829-1835, 2013.

14. Elmali N, Baysal O, Harma A, Esenkaya I and Mizrak B: Effects of resveratrol in inflammatory arthritis. Inflammation 30: 1-6, 2007.

15. El-Ghazaly MA, Nada AS, El-Hazek RM and Khayyal MT: Effect of selective COX-2 inhibitor, celecoxib on adjuvant-induced arthritis model in irradiated rats. Int J Radiat Biol 86: 1079-1087, 2010.

16. Chen XY, Li J, Cheng WM, Jiang H, Zhang L and Hu R: Effect of total flavonoids Chrysanthemum indicum on ultrastructure and secretory function of synoviocytes in adjuvant arthritis rats. Lat Am J Pharm 30: 2031-2036, 2011.
17. Yifan W, Dengming W, Zheng L, Yanping L and Junkan S: Triptolide inhibits CCR5 expressed in synovial tissue of rat adjuvant-induced arthritis. Pharmacol Rep 59: 795-799, 2007.

18. Bolon B, Morony S, Cheng Y, Hu YL and Feige U: Osteoclast numbers in Lewis rats with adjuvant-induced arthritis: identification of preferred sites and parameters for rapid quantitative analysis. Vet Pathol 41: 30-36, 2004.

19. Leech M, Xue JR, Dacumos A, Hall P, Santos L, Yang Y, Li M, Kitching AR and Morand EF: The tumour suppressor gene p53 modulates the severity of antigen-induced arthritis and the systemic immune response. Clin Exp Immunol 152: 345-353, 2008.

20. Chang Y, Wu Y, Wang D, Wei W, Qin Q, Xie G, Zhang L, Yan S, Chen J, Wang Q, Wu H, Xiao F, Sun W, Jin J and Wang W: Therapeutic effects of TACI-Ig on rats with adjuvant-induced arthritis via attenuating inflammatory responses. Rheumatology (Oxford) 50: 862-870, 2011.

21. Zheng FL, Chang Y, Jia XY, Huang M and Wei W: Effects and mechanisms of Cryptotanshinone on rats with adjuvant arthritis. Chin Med J (Engl) 124: 4293-4298, 2011.

22. Xuzhu G, Komai-Koma M, Leung BP, Howe HS, McSharry C, McInnes IB and $\mathrm{Xu}$ D: Resveratrol modulates murine collagen-induced arthritis by inhibiting Th17 and B-cell function. Ann Rheum Dis 71: 129-135, 2012.

23. Bevaart L, Vervoordeldonk MJ and Tak PP: Evaluation of therapeutic targets in animal models of arthritis: how does it relate to rheumatoid arthritis? Arthritis Rheum 62: 2192-2205, 2010.

24. Abdollahi-Roodsaz S, Joosten LA, Koenders MI, Devesa I, Roelofs MF, Radstake TR, Heuvelmans-Jacobs M, Akira S, Nicklin MJ, Ribeiro-Dias F and van den Berg WB: Stimulation of TLR2 and TLR4 differentially skews the balance of T cells in a mouse model of arthritis. J Clin Invest 118: 205-216, 2008.

25. Aizman E, Blacher E, Ben-Moshe O, Kogan T, Kloog Y and Mor A: Therapeutic effect of farnesylthiosalicylic acid on adjuvant-induced arthritis through suppressed release of inflammatory cytokines. Clin Exp Immunol 175: 458-467, 2014.

26. Song SS, Huang B, Wang QT, Wu YJ, Fu JJ, Zhang YF, Chang Y, Chen JY, Wu HX, Wang D, Zhang LL and Wei W: BF02, a recombinant TNFR2 fusion protein, alleviates adjuvant arthritis by regulating T lymphocytes in rats. Acta Pharmacol Sin 34: 414-423, 2013

27. Lichtenberger LM, Barron M and Marathi U: Association of phosphatidylcholine and NSAIDs as a novel strategy to reduce gastrointestinal toxicity. Drugs Today (Barc) 45: 877-890, 2009.

28. Half EE and Arber N: Chemoprevention of colorectal cancer: two steps forward, one step back? Future Oncol 2: 697-704, 2006.

29. Bjarnason I, Macpherson A, Rotman H, Schupp J and Hayllar J: A randomized, double-blind, crossover comparative endoscopy study on the gastroduodenal tolerability of a highly specific cyclooxygenase- 2 inhibitor, flosulide, and naproxen. Scand J Gastroenterol 32: 126-130, 1997.

30. Gheorghe KR, Thurlings RM, Westman M, Boumans MJ, Malmström V, Trollmo C, Korotkova M, Jakobsson PJ and Tak PP: Prostaglandin E2 synthesizing enzymes in rheumatoid arthritis B cells and the effects of B cell depleting therapy on enzyme expression. PLoS One 6: e16378, 2011.

31. Beavis PA, Gregory B, Green P, Cribbs AP, Kennedy A, Amjadi P, Palfreeman AC, Feldmann M and Brennan FM: Resistance to regulatory $\mathrm{T}$ cell-mediated suppression in rheumatoid arthritis can be bypassed by ectopic foxp3 expression in pathogenic synovial T cells. Proc Natl Acad Sci USA 108: 16717-16722, 2011

32. Nakayama H, Yaguchi T, Yoshiya S and Nishizaki T: Resveratrol induces apoptosis MH7A human rheumatoid arthritis synovial cells in a sirtuin 1-dependent manner. Rheumatol Int 32: 151-157, 2007. 